\title{
Unmoderated Posters: Urinary Incontinence
}

\author{
UP-59 \\ Evaluation of Three Penile Clamps for Post Prostatectomy Urinary \\ Incontinence \\ Martel, Tristan'; Bienz, Marc'; McCormack, Michael2; Karakiewicz, Pierre \\ I. ${ }^{2}$; Valiquette, Luc $^{2}$ \\ ${ }^{1}$ Université de Montréal, Montreal, QC, Canada; ${ }^{2}$ Centre Hospitalier de \\ I'Université de Montréal, Montreal, QC, Canada \\ Introduction and Objectives: Men with severe stress urinary incontinence \\ after a radical prostatectomy can use penile clamps either temporarily or \\ permanently. We developed a new incontinence clamp (Prototype) and in \\ this study we compared it to the two most commonly used commercial \\ clamps (Cunningham and Dribblestop) in order to assess its efficiency, \\ handling and comfort.
}

Methods: We did a prospective randomized block design clinical study. Eighteen patients with severe stress urinary incontinence after a radical prostatectomy were randomized and seventeen completed the study. Validated questionnaires (ICIQ-UI SF, IPSS, EORTC QLQ-PR25), non-validated questionnaires and weekly incontinence diaries were used to evaluate each patient over a period of 6 weeks. Weekly evaluations were done. Each patient tested each clamp in a randomized order for one week at home. Results: An improvement in incontinence ranging from significant to complete was found in $76.5 \%$ of subjects with the Cunningham, $82.4 \%$ with the Dribblestop and $29.4 \%$ with the prototype ( $p=0.01$ with Cochran). Using ICIQ-UI SF questionnaire, the median score without clamp was 17.0 [14.50-20.50], 12.0 [7.75-15.50] $(\mathrm{p}=0.024)$ with the Cunningham, 12.0 [9.0-15.0] $(p=0.002)$ with the Dribblestop and 13.0 [11.00-15.50] $(p=0.07)$ with the proptotype. Subjects identified as their preferred choice the Dribblestop $(41.2 \%)$, the Cunningham $(35.3 \%)$ and the prototype at $(23.5 \%)(p=0.662)$. Only non-parametric tests were used.

Conclusions: Penile clamps improved severe stress urinary incontinence after a radical prostatectomy. The frequency of incontinence and amount of urine loss were lower with the three tested clamps. The Dribblestop was the preferred clamp in our study.

\section{UP-60}

\section{Clinical Research: Prospective Evaluation of Pain during Botilum} Toxin A Detrusor Injection

St-Pierre, Rachel; Marceau-Grimard, Maryse; Moore, Katherine

Université Laval, Quebec, QC, Canada

Introduction and Objective: Botulinum toxin A (BTX-A) detrusor injection has been approved in 2011 by the FDA for the treatment of neurogenic detrusor overactivity. We aim to evaluate the level of pain during the different steps of BTX-A detrusor injections.

Methods: Between July and October 2013, we offered allour patients scheduled for BTX-A injections entry into our prospective study. During the standard procedure, the patients were asked to grade their level of pain according to a visual analogic scale 0-10 (VAS) at five different moments: arrival, urethral insertion, bladder filling, BTX-A injections and five minutes after removal of the instruments.

Results: Injections were performed in 36 patients (18 men, 18 women) with spinal cord injury $(\mathrm{SCl})(69 \%)$, multiple sclerosis (MS) $(22 \%)$, familial spastic paraparesis (FSPP) $(6 \%)$, Huntington disease (HD) $(3 \%)$, myelomeningocele (MMC) (3\%). In patients with complete $\mathrm{SCl}$ and $\mathrm{MMC}$, injections were not adding more discomfort to the cystoscopy. For patients with MS and HD, cystoscopy had little impact on pain level compared to the injections (mean differential pain: $2 \mathrm{v} / \mathrm{s} 4,3$ ). In patients with incomplete $\mathrm{SCl}$, urethral manipulation seemed more painful with non-significant elevation of pain with BTX-A injections (mean differential pain: $3,8 \mathrm{v} / \mathrm{s} 1,4)$. The mean differential pain in patients with complete cervical (CCSCl) and dorsal $\mathrm{SCl}$ (CDSCl), incomplete dorsal SCI (IDSCI) and MCC were less than 1.

Conclusions: In patients with complete $\mathrm{SCl}$ and $\mathrm{MMC}$, pain associated with the procedure was low. With incomplete $\mathrm{SCl}$, pain can be better attributed to urethral manipulation than actual detrusor injections. For MS, FSPP and HD patients, detrusor injections explained their pain but their pain threshold seemed lower than the $\mathrm{SCl}$ population during urethral manipulation. Our goal now is to develop better strategies to minimize painful stimuli in this recurrent procedure.

\section{UP-61}

An In-depth Psychological Analysis of Three Descriptive Accounts of Urinary Urgency in Incontinent Women

Miceli, Paula ${ }^{1}$; Love, William²; Radomski, Sidney ${ }^{3}$; Giorgi, Amedeo ${ }^{4}$; Katz, Joel $^{1}$

${ }^{1}$ York University, Toronto, ON, Canada; ${ }^{2}$ Private Practice, Burlington, ON, Canada; ${ }^{3}$ University Health Network, Toronto, ON, Canada; ${ }^{4}$ Saybrook University, San Francisco, CA, United States

Introduction and Objectives: Patient-practitioner communication is an important issue in the assessment of Overactive Bladder Syndrome (OABS). The present study sought to better understand the meaning of urgency in incontinent women to enhance communication in clinical settings.

Method: Ten ambulatory female patients with a diagnosis of OABS or mixed urinary incontinence (UI) with clinically significant urgency were recruited (mean (SD) age: $68 \pm 7.2 \mathrm{y}$; range: $56-77 \mathrm{y}$ ). Interview questions elicited detailed descriptions of situations in which participants experienced urgency. The analytic procedure followed the method of Giorgi (2009; The Descriptive Phenomenological Method in Psychology, Duquesne Press). Results: Given the depth of analysis, three transcripatients were randomly selected (ages: $58 \mathrm{y}, 72 \mathrm{y}, 74 \mathrm{y}$ ), which included 14 descriptions of urgency, 10 of which included urge or stress leakage. Urgency was acute and episodic. All episodes featured the participants' situational awareness of their incapability to contain urine, as well as a sense of pressure and uncertainty with respect to reaching a toilet. Irrespective of whether leakage ensued, all descriptions were characterized by emotional sequelae related to the disclosure of urinary flow to others (i.e., distress, embarrassment, sense of inadequacy), which the women attempted to alleviate using material, psychological, relational, and medical strategies. When access to toilets was hindered by idiographic health and environmental circumstances, the emotional impact was intensified.

Conclusions: Incontinent women perceived urgency as a loss of capability to contain urine that had negative social and emotional consequences. These results suggest that practitioner communications about urgency may be enhanced with attention to its scope of impact rather than its biological mechanisms (e.g., as an abnormal desire to void) 